Michael Scheffler

Management groß angelegter Grundstücksentwässerungsanlagen

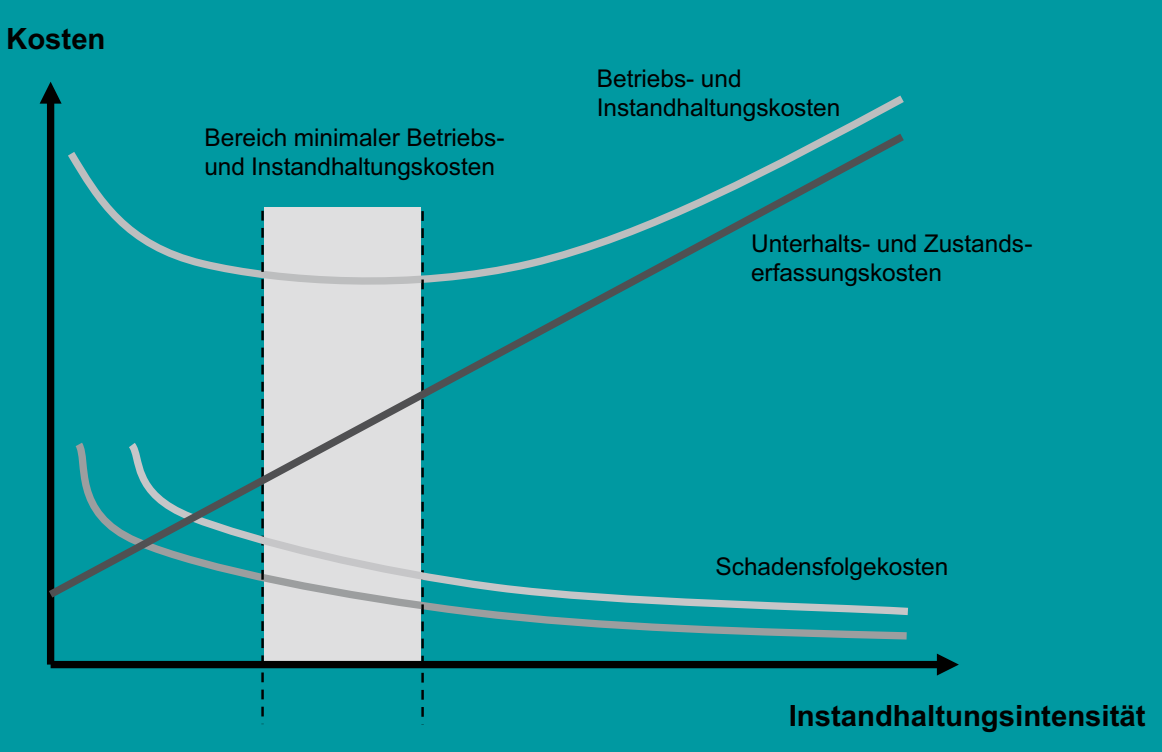

Fraunhofer IRB Verlag 
Michael Scheffler

Management groß angelegter Grundstücksentwässerungsanlagen 
Mit großer Freude widme ich dieses Buch meiner lieben Tochter Fiona, der ich auf ihrem weiteren Lebensweg besonnenen Mut, geistige Freiheit und einen sicheren Blick für die wirklich wichtigen Dinge in einer friedlichen Zeit wünsche. 
Michael Scheffler

Management groß angelegter Grundstücksentwässerungsanlagen

Organisation und Optimierung von Betrieb und Instandhaltung - ein Handbuch

Fraunhofer IRB Verlag 
Bibliografische Information der Deutschen Nationalbibliothek

Die Deutsche Nationalbibliothek verzeichnet diese Publikation in der Deutschen Nationalbibliografie; detaillierte bibliografische Daten sind im Internet über <http://dnb.d-nb.de> abrufbar.

ISBN (Print): 978-3-8167-8537-8

ISBN (E-Book): 978-3-8167-8870-6

Redaktion: Nicole Herr, Fraunhofer IRB Verlag

Umschlaggestaltung: Martin Kjer, Fraunhofer IRB Verlag

Herstellung: Katharina Kimmerle, Fraunhofer IRB Verlag

Satz: Fotosatz Buck, Kumhausen/Hachelstuhl

Druck: freiburger graphische betriebe $\mathrm{GmbH} \&$ Co. KG, Freiburg

Für den Druck des Buches wurde chlor- und säurefreies Papier verwendet.

Alle Rechte vorbehalten

Dieses Werk ist einschließlich aller seiner Teile urheberrechtlich geschützt. Jede Verwertung, die über die engen Grenzen des Urheberrechtsgesetzes hinausgeht, ist ohne schriftliche Zustimmung des Fraunhofer IRB Verlages unzulässig und strafbar. Dies gilt insbesondere für Vervielfältigungen, Übersetzungen, Mikroverfilmungen sowie die Speicherung in elektronischen Systemen.

Die Wiedergabe von Warenbezeichnungen und Handelsnamen in diesem Buch berechtigt nicht zu der Annahme, dass solche Bezeichnungen im Sinne der Warenzeichen- und Markenschutz-Gesetzgebung als frei zu betrachten wären und deshalb von jedermann benutzt werden dürften.

Sollte in diesem Werk direkt oder indirekt auf Gesetze, Vorschriften oder Richtlinien (z. B. DIN, VDI, VDE) Bezug genommen oder aus ihnen zitiert werden, kann der Verlag keine Gewähr für Richtigkeit, Vollständigkeit oder Aktualität übernehmen. Es empfiehlt sich, gegebenenfalls für die eigenen Arbeiten die vollständigen Vorschriften oder Richtlinien in der jeweils gültigen Fassung hinzuzuziehen.

Der Autor übernimmt keinerlei Gewähr für die Aktualität, Korrektheit, Vollständigkeit oder Qualität der bereitgestellten Informationen. Haftungsansprüche gegen den Autor, welche sich auf Schäden materieller oder ideeller Art beziehen, die durch die Nutzung oder Nichtnutzung des Handbuches bzw. durch die Nutzung fehlerhafter und unvollständiger Informationen verursacht wurden, sind grundsätzlich ausgeschlossen, sofern seitens des Autors kein nachweislich vorsätzliches oder grob fahrlässiges Verschulden vorliegt.

(C) by Fraunhofer IRB Verlag, 2013

Fraunhofer-Informationszentrum Raum und Bau IRB

Nobelstr. 12, 70569 Stuttgart

Telefon 0711 970-2500

Telefax 0711 970-2508

E-Mail irb@irb.fraunhofer.de

http://www.baufachinformation.de 


\section{Vorwort}

In den letzten Jahren ist ein ganzes Spektrum an Aspekten zur Daseinsvorsorge in politischen Diskussionen in den Vordergrund gerückt - die geordnete Abwasserentsorgung in der Stadtentwässerung hat dabei unzweifelhaft an Bedeutung gewonnen. Das zeigt sich nicht nur allein daran, dass im öffentlichen Bereich für die Reinigung, die Zustandserfassung und die Sanierung von Entwässerungsanlagen nach wie vor erhebliche Bemühungen und Aufwendungen erkennbar sind und das Ziel verfolgt wird, die Betriebsfähigkeit der öffentlichen Abwasseranlagen dauerhaft zu sichern. Auch Grundstücksentwässerungsanlagen (im Weiteren GE-Anlagen genannt) rücken landesweit zunehmend in das Blickfeld. Der flächendeckend schlechte bauliche Zustand bestehender GE-Anlagen wird in den kommenden Jahren gewaltige Investitionen für detaillierte Bauzustandsanalysen, für die Dokumentation von Inspektionsergebnissen, für hochwertige Sanierungsmaßnahmen sowie für instandhaltungsfreundliche Konzeptionen erfordern.

Anforderungen an größere GE-Anlagen, etwa auf gewerblich oder industriell genutzten Grundstücken, sind besonders anspruchsvoll. Hier haben Betreiber über die Regelaufgaben für Betrieb und Unterhalt hinaus Aufgaben wahrzunehmen, die die Ergreifung von rein technischen Maßnahmen weit übertreffen. So sind mit zunehmender GE-Anlagengröße organisatorische und strukturelle Aspekte in den Blick zu nehmen, damit wirtschaftliche und rechtskonforme GE-Anlagenbetriebe gewährleistet werden können. Im Mittelpunkt stehen dabei meist systematisierte und koordinierte Umsetzungen von Instandhaltungsmaßnahmen, aber auch die Hebung betriebs- und arbeitsorganisatorischer Optimierungspotenziale, hinterlegt mit Prozessdarstellungen. Um die damit verbundenen Aufgaben und Belastungen in Grenzen zu halten, gilt es, den Anspruch eines wirtschaftlich und technisch umsichtigen Handelns im Auge zu behalten, der verlangt, dass wirtschaftliche und technische Ziele vor dem Hintergrund rechtlicher Vorgaben miteinander vereinbar sein müssen.

Damit alle Anforderungen, denen sich GE-Anlagenbetreiber heute zu stellen haben auch zu gewünschten Ergebnissen führen, ist es notwendig auf fundierte und hilfreiche Grundlagen zurückgreifen zu können. Dazu wurde das vorliegende Handbuch erarbeitet.

Die Erarbeitung eines Handbuches zur Optimierung und Organisation von Betrieb und Instandhaltung großer GE-Anlagen, welches leichtverständlich formuliert ist und das sich auf der Höhe der Fachdiskussionen befindet, ist für sich allein genommen schon ein hoher Anspruch, der nur schwer zu erfüllen ist. Die Anforderungen und Aufgaben im Betrieb und in der Instandhaltung von GE-Anlagen dann aber noch so vollständig abzubilden, dass alle As- 
pekte für jeden Einzelfall abgedeckt werden, ist kaum zu erreichen. Selbst mit einem inhaltsreichen Handbuch wie dem vorliegenden, lassen sich nicht alle individuellen Fragestellungen beantworten. Vertiefende Betrachtungen spezifischer Gesichtspunkte würden zwar Unmengen an Informationen mit sich bringen. Sie würden aber nicht zum Vorteil allgemeiner betrieblicher Akzeptanz und Anwendbarkeit geraten können. Aufgrund der Komplexität der Grundstücksentwässerung sind letztlich immer Einzelfallbetrachtungen erforderlich, die in ihrer Analyse stets unterschiedlich ausfallen.

Mit der vorliegenden Publikation wird ein auf diese Situation abgestimmter Weg beschritten. Das Handbuch ist außerordentlich breit angelegt und orientiert sich auf der Grundlage zahlreicher Fachdiskussionen und meiner langjährigen berufspraktischen Erfahrungen, die ich als Gutachter, Planer und Leiter bei der Umsetzung von Projekten der Wasserversorgungs- und Abwassertechnik sammeln konnte, an häufigen Problemstellungen sowie am gegenwärtigen Informationsdefizit bei Eigentümern großer GE-Anlagen. Ich bin davon überzeugt, dass durch die Verwendung eines geeigneten Führungshilfsmittels (das heißt hier mit einem eigens auf den Betrieb und die Instandhaltung großer GE-Anlagen ausgelegten Handbuch, in dem die Grundzüge zur Einführung eines spezifischen prozessorientierten Managementsystems ausführlich behandelt werden) eine gewinnbringende Unterstützung im Hinblick auf Kosten-Nutzen-Verhältnisse, auf Aufgabenkoordinationen und auf organisatorische Belange zur Verfügung gestellt wird, über das spezifische Verflechtungen zwischen strategischen Überlegungen und operativen Leistungen besser erkennbar und umsetzbar sind.

Gegenstand des Handbuches sind technische und organisatorische Vorgänge zur Herstellung und dauerhaften Sicherung der Funktions- und Betriebsfähigkeit von großen GE-Anlagen bebauter Grundstücke, wobei das Management sowie die prozessorientierte Umsetzung und Abwicklung von Betriebs- und Instandhaltungsaufgaben einen Themenschwerpunkt bilden. Die ökologische Komponente liegt dabei in der Schaffung eines Rüstzeugs für Betreiber großer GE-Anlagen zur Vermeidung von Beeinträchtigungen der Umweltkompartimente Boden und Grundwasser und zur Erhaltung ihrer ökologischen Funktionen. 
Die Kernaussagen des Handbuches mögen für viele Einzelfälle direkt zutreffend sein. Dennoch bedarf es zur langfristigen Sicherstellung erwünschter betrieblicher Optimierungseffekte und sachgerechter Abwicklungen von Instandhaltungsmaßnahmen innerbetrieblicher regelmäßiger Überprüfungen, Anpassungen und Aktualisierungen. Daher sind die zur Verfügung gestellten Inhalte des Handbuches stets auf den individuellen Bedarf hin abzustellen und mit spezifischen rechtlichen Rahmenbedingungen abzugleichen.

Michael Scheffler, Kassel, im Herbst 2012 


\title{
Verzeichnis der Abkürzungen
}

\author{
Kürzel \\ a.a.R.d.T \\ $\mathrm{AbwV}$ \\ $\mathrm{AHO}$ \\ ATV \\ DVWK \\ Bedeutung \\ allgemein anerkannte Regeln der Technik \\ Verordnung über Anforderungen an das Einleiten von \\ Abwasser in Gewässer (Abwasserverordnung) \\ Ausschuss der Ingenieurverbände und Ingenieurkam- \\ mern für die Honorarordnung e.V. \\ Abwassertechnische Vereinigung e.V. (bis 31.12.1999) \\ Deutscher Verband für Wasserwirtschaft und Kulturbau \\ e.V. (bis 31.12.1999) \\ B \\ Beton \\ BauO NRW Bauordnung für das Land Nordrhein-Westfalen \\ BayWG \\ BbgWG \\ Bayerisches Wassergesetz \\ BBodSchG \\ Brandenburgisches Wassergesetz \\ $\mathrm{BGH}$ \\ Bundes-Bodenschutzgesetz \\ BImSchG \\ Bundesgerichtshof \\ BL \\ Bundes-Immisionsschutzgesetz \\ BMFT \\ Bereichsleiter \\ BrWG \\ Bundesministerium für Forschung und Technik \\ $\mathrm{BuU}$ \\ Bremisches Wassergesetz \\ BVB \\ BWG \\ Betriebs- und Unterhaltsbuch \\ Besondere Vertragsbedingungen \\ BWWG \\ Berliner Wassergesetz \\ CAFM-Systeme \\ CEN \\ Baden-württembergisches Wassergesetz \\ Computer Aided Facility Management-Systeme \\ Comité Européen de Normalisation, European Com- \\ mittee for Standardization, Europäisches Komitee für \\ Normung \\ DIBT \\ Deutsches Institut für Bautechnik \\ DIGEA \\ Dienstleister für die Instandhaltung von GE-Anlagen \\ DIN \\ Deutsches Institut für Normung e. V. \\ $\mathrm{DN}$ \\ Nennweite, die annähernd dem Zahlenwert der Lichten \\ Weite in Millimeter entspricht \\ DVGW Deutsche Vereinigung des Gas- und Wasserfaches e.V. \\ DWA Deutsche Vereinigung für Wasserwirtschaft, Abwasser \\ und Abfall e.V. (ab 01.01.2000 durch Zusammenschluss \\ von ATV und DVWK) \\ eAN Externer Auftragnehmer \\ eID Externer Ingenieurdienstleister \\ EigenkontrollVO Eigenkontrollverordnung
}




\begin{tabular}{|c|c|}
\hline EigÜVO & Eigenüberwachungsverordnung \\
\hline EKVO & Eigenkontrollverordnung \\
\hline EMR & ElektroMagnetische Reflexion \\
\hline EP & Epoxidharz \\
\hline EU & Europäische Union \\
\hline EU WRRL & Europäische Wasserrahmenrichtlinie \\
\hline EÜV & Eigenüberwachungsverordnung \\
\hline EÜVOA & $\begin{array}{l}\text { Landesverordnung über die Eigenüberwachung von } \\
\text { Abwasseranlagen }\end{array}$ \\
\hline EWG & Europäische Wirtschaftsgemeinschaft \\
\hline EWS & Entwässerungssatzung \\
\hline FBS & Fachvereinigung Betonrohre und Stahlbetonrohre e.V. \\
\hline FM & Facility-Management \\
\hline Fz & Faserzement \\
\hline GE-Anlage & Grundstücksentwässerungsanlage \\
\hline EFMA & $\begin{array}{l}\text { German Facility Management Association - Deutscher } \\
\text { Verband für Facility-Management e.V. }\end{array}$ \\
\hline & Glasfaserverstärkter Kunststoff \\
\hline & Grundgesetz der Bundesrepublik Deutschland \\
\hline L/UL & Geschäftsleitung/Unternehmensleitung \\
\hline $\mathrm{DB}$ & Hauptverband der Deutschen Bauindustrie \\
\hline HD-Spülung & Hochdruck-Spülung \\
\hline mbAbwG & Hamburgisches Abwassergesetz \\
\hline HOAI & $\begin{array}{l}\text { Verordnung über die Honorare für Leistungen der Archi- } \\
\text { tekten und Ingenieure (Honorarordnung für Architekten } \\
\text { und Ingenieure) }\end{array}$ \\
\hline & Honorarzone der HOAI \\
\hline WG & Hessisches Wassergesetz \\
\hline & Immobilien-Facility-Management \\
\hline & Infektionsschutzgesetz \\
\hline & Institut für Kanalisationstechnik, Gelsenkirchen \\
\hline IVU-Richtlinie & $\begin{array}{l}\text { Integrierte Vermeidung und Verminderung der Umwelt- } \\
\text { verschmutzung- Richtlinie }\end{array}$ \\
\hline $\mathrm{k}_{\mathrm{f}}$-Wert & Wasserdurchlässigkeitsbeiwert in m/s \\
\hline KNA & Kosten-Nutzen-Analyse \\
\hline KRV & Kunststoffrohrverband e.V. \\
\hline rW-/AbfG & Kreislaufwirtschafts- und Abfallgesetz \\
\hline & (dynamische) Kostenvergleichsrechnung \\
\hline NA & Kostenwirksamkeitsanalyse \\
\hline WA & Länderarbeitsgemeinschaft Wasser \\
\hline & Leistungsbeschreibung \\
\hline
\end{tabular}




$\begin{array}{ll}\text { LBO NRW } & \text { Landesbauordnung Nordrhein-Westfalen } \\ \text { LBuI } & \text { Leiter Betrieb und Instandhaltung } \\ \text { LfU BW } & \text { Landesanstalt für Umweltschutz Baden-Württemberg } \\ \text { LGA } & \text { Landesgewerbeanstalt Bayern } \\ \text { LV } & \text { Leistungsverzeichnis } \\ \text { LwaG } & \text { Wassergesetz des Landes Mecklenburg-Vorpommern } \\ \text { LWG } & \text { Landeswassergesetz } \\ \text { LWG NRW } & \text { Landeswassergesetz Nordrhein-Westfalen } \\ \text { MA } & \text { Mitarbeiter } \\ \text { MBO } & \text { Musterbauordnung } \\ \text { MULF } & \text { Hessisches Ministerium für Umwelt, Landwirtschaft } \\ & \text { und Forsten } \\ \text { MURL } & \text { Ministerium für Umwelt Raumordnung und Landwirt- } \\ & \text { schaft des Landes Nordrhein-Westfalen } \\ \text { mWs } & \text { Meter Wassersäule (1,00 mWs = 10kPa = 0,1 bar = 0,1 at) } \\ \text { NAW } & \text { Normenausschuss Wasserwesen im DIN } \\ \text { NdsWG } & \text { Niedersächsisches Wassergesetz } \\ \text { NWA } & \text { Nutzwertanalyse } \\ \text { oAK } & \text { Objektspezifisches Abwasserkonzept gemäß 4-EIP } \\ \text { ÖBü } & \text { Örtliche Bauüberwachung } \\ \text { PE } & \text { Projektentscheid } \\ \text { PE-HD } & \text { Polyethylen hoher Dichte } \\ \text { pH-Wert } & \text { Konzentration von Wasserstoffionen in wässrigen } \\ & \text { Lösungen } \\ \text { PKBW } & \text { Projektkostenbarwert } \\ \text { PP } & \text { Polypropylen } \\ \text { PU } & \text { Polyurethan } \\ \text { PVC-U } & \text { Polyvinylchlorig weichmacherfrei } \\ \text { R.d.T. } & \text { Regeln der Technik } \\ \text { RSV } & \text { Rohrleitungsanierungsverband e.V. } \\ \text { RÜB } & \text { Regenüberlaufbecken } \\ \text { RW-Kanal } & \text { Regenwasserkanal } \\ \text { S.d.T. } & \text { Stand der Technik } \\ \text { S.v.W.u.T. } & \text { Stand von Wissenschaft und Technik } \\ \text { SächsWG } & \text { Sächsisches Wassergesetz } \\ \text { StGB } & \text { Strafgesetzbuch } \\ \text { StLB } & \text { Standardleistungsbuch } \\ \text { Stz } & \text { Steinzeug } \\ \text { SÜVO, SüVO, } & \\ \text { SüwV } & \text { Selbstüberwachungsverordnung } \\ & \end{array}$


SüwV Kan Verordnung zur Selbstüberwachung von Kanalisationen und Einleitungen von Abwasser aus Kanalisationen im Mischsystem und im Trennsystem (Selbstüberwachungsverordnung Kanal) von Nordrhein-Westfalen

SWG Saarländisches Wassergesetz

TGM Technisches Gebäudemanagement

ThürAbwEKVO Thüringer Abwassereigenkontrollverordnung

ThürWG

TL Thüringer Wassergesetz

TM Teamleiter

TöB

UVM BW

Teammitarbeiter

Träger öffentlicher Belange

Ministerium für Umwelt und Verkehr Baden-Württemberg

UVP

Umweltverträglichkeitsprüfung

UVV

Unfallverhütungsvorschrift

VDI

Verband Deutscher Ingenieure

VDMA

VNW

$\mathrm{VOB}$

Verband Deutscher Maschinen- und Anlagenbau e.V.

Verband Norddeutscher Wohnungsunternehmen e.V.

$\mathrm{VOB} / \mathrm{A}$

Vergabe- und Vertragsordnung für Bauleistungen

$\mathrm{VOB} / \mathrm{B}$

Vergabe- und Vertragsordnung für Bauleistungen, Teil A

$\mathrm{VOF}$

VOL Vergabe- und Vertragsordnung für Bauleistungen, Teil B Verdingungsordnung für freiberufliche Leistungen Verdingungsordnung für Leistungen

VV

WG Verwaltungsvorschrift

Wassergesetz

WGLSA

WGLSH

Wassergesetz des Landes Sachsen-Anhalt

WHG Wassergesetz des Landes Schleswig-Holstein Gesetz zur Ordnung des Wasserhaushalts (Wasserhaushaltsgesetz)

wMP Weitere Maßnahmenpauschale

WR

ZB

Wirtschaftlichkeitsrechnung

Zentralbereich

$z M P$

Zusätzliche Maßnahmenpauschale 


\section{Inhalt}

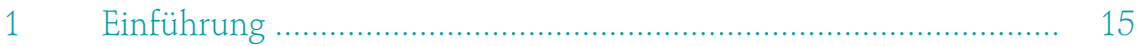

1.1 Aktuelle Situation und Veranlassung …………........................... 18

1.2 Nutzen und Aufbau der Publikation ............................................ 22

2 Grundlagen des Betriebes und der Instandhaltung von GE-

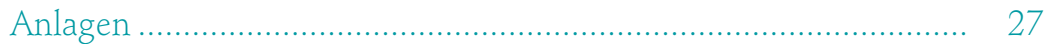

2.1 Gesetzliche Rahmenbedingungen ............................................. 27

2.2 Technische Standards ............................................................ 36

2.3 Begriffsstruktur von Betrieb und Instandhaltung ........................ $\quad 42$

$2.4 \quad$ Ziele und Strategien der Instandhaltung ...................................... 46

2.5 Betrieb und Instandhaltung - Grundsätze und Empfehlungen ..... 59

2.6 Systembedingungen und Grenzen bei der erstmaligen

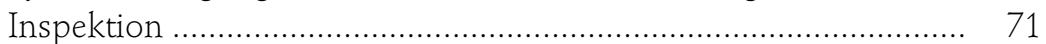

2.7 Stufenübersicht der erstmaligen Instandhaltung von bestehenden

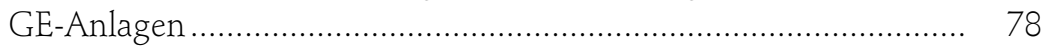

$3 \quad$ Konzeption einer praxisnahen Vorgehensweise bei der erstmaligen Instandhaltung - 4-EbenenInstandhaltungsprogramm (4-EIP) ........................................... 95

3.1 Einführung in das 4-EIP ....................................................... 96

3.2 Ebene 1: Bestands- und Funktionsanalyse …………………........ 99

3.3 Ebene 2: Objektspezifisches Abwasserkonzept .......................... 103

3.4 Ebene 3: Reinigung, Zustandserfassung und -auswertung .......... 110

3.5 Ebene 4: Sanierung ............................................................. 121

4 Aufbauorganisation einer Betriebs- und Instandhaltungsabteilung ..................................................... 131

4.1 Personalplanung und -qualifikation ………………………...... 133

4.2 Arbeits- und Gesundheitsschutz ........................................... 140

4.3 Aufgaben und Pflichten des Betreibers und anderer Beteiligter ... 145

4.4 Besondere Aspekte bei Planung, Bau und Betrieb ...................... 158

4.5 Aufgaben und Funktion einer Betriebs- und

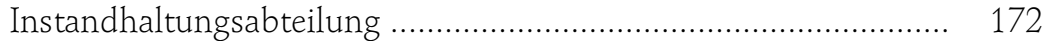

4.6 Dienst- und Betriebsanweisung ………………...................... 175

5 Ablauforganisation im Regelbetrieb - Technisches Betriebs- und Instandhaltungsmanagement ................................................ 181

5.1 Strategisches und operatives Betriebs- und Instandhaltungsmanagement

5.2 Störfallbearbeitung/Bereitschaftsdienst ..................................... 189 
5.3 Dokumentation von GE-Anlagendaten in einem GE-

Anlagenkataster

5.4 Entscheidungsprozess zur Findung effizienter Lösungen im

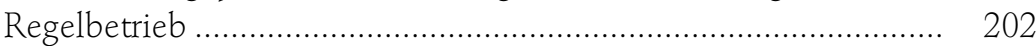

5.5 Erstellung und Fortschreibung von Prozessdokumenten ............ 214

$6 \quad$ Grundzüge eines Instandhaltungscontrollings .......................... 225

6.1 Technisch-wirtschaftliches Instandhaltungscontrolling ............... 225

6.2 Aspekte der Kostenrechnung ……………............................... 227

6.3 Betriebs- und Instandhaltungskosten ........................................ 232

6.4 Hinweise zur Konzeption von Kennzahlen .............................. 241

$7 \quad$ Projektmanagement bei Betrieb und Instandhaltung .................. 247

7.1 Typische Projektmerkmale ......................................................... 249

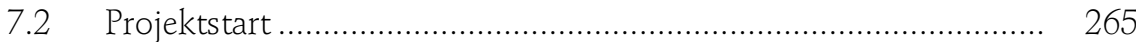

7.3 Projektplanung ...................................................................... 267

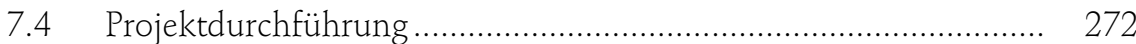

7.5 Projektabschluss ................................................................. 275

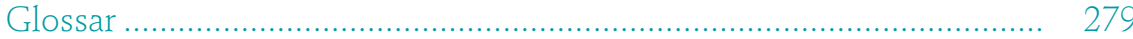

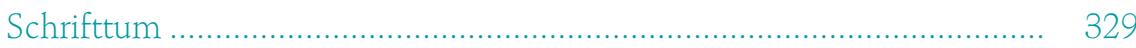

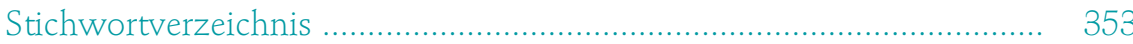

Anhang I bis IV auf CD-ROM 